\title{
New Editor-in-Chief
}

\author{
Adriaan M. H. van der Veen ${ }^{1}$
}

Published online: 16 January 2018

(C) Springer-Verlag GmbH Germany, part of Springer Nature 2018

When Steffen Pauly, Editorial Director at Springer Nature, approached me as candidate for the post of Editor-in-Chief, my first reaction was that if I would ever consider such as position, it would be for this journal, which I have known so well since it was founded. For over 25 years, I have been active in metrology research and development, regular calibration and testing work, accreditation assessments and standardisation. This practical experience is paired with an established track record in publishing scientific results, managing projects, running proficiency tests and giving training courses.

Accreditation and Quality Assurance (ACQUAL) fulfils an important role in disseminating innovations and best practices in chemical measurement. Contributions to the journal thereby often connect aspects of metrology, standardisation and accreditation. As measurement in chemistry is still rapidly developing to meet todays and tomorrows societal needs, there is a bright future for the journal. Many of the developments happen nowadays in networks, be it ISO (International Organization for Standardization), CCQM (Consultative Committee for Amount-of-Substance), ILAC (International Laboratory Accreditation Organisation), among many others. Programmes, such as the European Metrology Research Programme and the European Metrology Programme for Innovation and Research, contribute significantly to the proliferation and further development of metrological concepts, also in new areas. ACQUAL has positioned itself over the years as the journal that informs the fields about novel developments, new ideas and everything else relevant to experts and practitioners in chemical measurement.

The publication of ISO 17034:2016 and ISO/IEC 17025:2017 will make reference material producers, calibration and testing laboratories think about the systems they operate to demonstrate competence. The new standards move away from specifying exactly what needs to be documented to a more risk-based approach, which calls for innovative solutions to meet these new demands. Traditionally, the

\footnotetext{
Adriaan M. H. van der Veen

avdveen@acqual.org

1 Rijsbergen, The Netherlands
}

publication of such new standards leads to papers highlighting key changes and their impact, followed by papers describing competent applications and implementations.

Also in other areas there are developments that will change our view on measurement in the near future. Two examples from the Joint Committee on Guides in Metrology concern the standardisation and harmonisation of the vocabulary for "qualitative measurement" and the embracement of the principles of Bayesian statistics in the evaluation of measurement uncertainty. One of the key issues debated with respect to the International Vocabulary of Metrology is whether "measurement" should also be applied to properties not being quantities, or that a different term is needed. Such decisions have far-reaching consequences and are therefore energetically debated.

The proliferation of more advanced methods in evaluating measurement uncertainty and measurement data, for example, those based on the Monte Carlo methods from Supplement 1 to the Guide to the expression of Uncertainty in Measurement (GUM-S1), are more demanding to the practitioner, yet at the same time much more flexible than the law of propagation of uncertainty. Especially in situations where the traditional approach of propagating uncertainties fails, the Monte Carlo method from GUM-S1 and Bayesian methods may be of assistance to the chemist, notwithstanding that the use of such methods implies different prerequisites. Making these methods better accessible requires paving the path, for example, by providing and documenting competent applications of these methods for problems.

In my new role, together with Ales Fajgelj, it is a privilege to contribute to the expansion of the journal's role and reputation in the field. Existing collaborations will be maintained and new ones be developed, to ensure that developments in science, standardisation and accreditation in chemical measurement reach the entire field, thus playing a key role in "creating impact". Last but not least, I would like to thank our predecessors, Editors-in-Chief Heiner Korte and Hendrik Emons for their efforts and keeping the journal so well in shape.

\section{Adriaan van der Veen \\ Editor-in-Chief}

